Wolf-Rayet Phenomena in Massive Stars and Starburst Galaxies

Proceedings IAU Symposium No. 193, (C)1999 IAU

K.A. van der Hucht, G. Koenigsberger \& P.R.J. Eenens, eds.

\title{
Some aspects of the spectral variability of WR 141
}

\author{
Millen M. Ivanov, Leonid N. Georgiev ${ }^{1}$ \\ Department of Astronomy, Sofia University, Bulgaria
}

T.S. Valchev

Institute of Astronomy, Bulgarian Academy of Sciences, Bulgaria

B.V. Efremova, and Svetozar A. Zhekov ${ }^{2}$

Space Research Institute, Bulgarian Academy of Sciences, Bulgaria

\begin{abstract}
An analysis of the behaviour of the He II 5411 line wings is presented. The spectral classification of the companion is discussed on the basis of a simple geometric model of the colliding stellar winds zone.
\end{abstract}

\section{Introduction and observations}

Up to recently, WR 141 was one of the least investigated northern Wolf-Rayet binaries. Hiltner (1945) has published the first orbital solution and has proposed $P=21.64$ d. During the last 50 years his ephemeris was accepted (or slightly modified) in the predominant studies of WR 141 (see the overview of Ivanov et al. 1999, hereafter I99). Combining all radial-velocity (RV) measurements available in the literature with new high-resolution data, Marchenko et al. (1998; hereafter M98), and independently I99, derived improved orbital elements of the system with $P=21.6895 \mathrm{~d} \pm 0.0003 \mathrm{~d}$ (M98) or $P=21.6878 \mathrm{~d} \pm 0.0001 \mathrm{~d}$ (I99), respectively. In addition, M98 found O5 to be the most probable spectral class of the secondary. Taking into consideration these results, the aim of this study was to examine the phase-dependence of the global spectral variations, typical for WR binaries, and to investigate the influence of the O-star companion on the shapes of the spectral lines.

High-resolution spectroscopic observations were carried out at the 2-m RCC telescope of the BNAO-Rozhen during June 1996-March 1998. The Bausch \& Lomb grating with $632 \mathrm{~mm}^{-1}$ was used to reach a dispersion of $0.2 \AA / \mathrm{pix}$ at the He II 5411 line. In addition, we have obtained some echelle spectra at the OAN in San Pedro Martir, Mexico, during September 1996 and August 1997. The spectra were reduced with the standard IRAF packages and, for comparison, were re-binned to equal dispersion of $0.2 \AA / \mathrm{pix}$.

${ }^{1}$ present address: Instituto de Astronomia, UNAM, Mexico

${ }^{2}$ present address: JILA, University of Colorado, USA 


\section{Results}

\subsection{Global profile variations}

An examination of the behaviour of the line-wings of He II 5411 was performed after corrections for the RV variations. The wavelength positions of the red $\left(\lambda_{r}\right)$ and blue $\left(\lambda_{b}\right)$ wing were measured using a chord on level 1.75 above the continuum. Surprisingly, we found a strong phase-dependency of these measurements. And what is more, the changes of the blue and red wing are simultaneous: on the plot $\left(\lambda_{\mathrm{r}}\right)$ vs. $\left(\lambda_{\mathrm{b}}\right)$ the slope coeficient is -0.96 . Since we work with normalized fluxes, this effect could be related to the phase-locked changes of the continuum flux, induced by the orbital motion of the companion. All the spectra, when arranged according to the I99 phase, support this conclusion.

\subsection{Effects of the colliding winds}

We tried to find out whether the variability in the He I 5876 line-profile could be due to the orbital motion of the colliding stellar winds (CSW) zone, occuring around the $\mathrm{O}$-star companion. This zone is extremely ionized and is not emitting at optical wavelenghts, but at different orbital phases it will diminish emission in the He I 5876 line at different radial velocities (simply because part of the WR wind will be missing due to the shock cone). If so, we can expect to see part of the line to have lower emission - something like a 'gap' in the emissionline profile - and the position of this 'gap' to be phase-dependent. Intending to obtain a rough evaluation of the aforementioned effect, a simple geometrical model was made, assuming the line to be optically thin, the velocity of the WR wind to be constant with $v=1700 \mathrm{~km} \mathrm{~s}^{-1}$, and the line-forming region to be between 6 and 40 stellar radii (Hillier 1987). If the companion star is of type O9V (Lamontagne et al. 1996), the effect of diminution of the emission caused by the CSW region could not explain the variability of He I 5876 line profile: at phase 0.95 the maximal depth of the 'gap' is only $1.3 \%$ of the full emission. The case of OV5-type stars (M98) is more probable. At the same phase, we expect the emission in the 'gap' to be $12 \%$ lower than in the rest of the line. So if the variability of He I 5876 line profile is due to the presence of the CSW region, then the secondary in WR 141 should most likely be classified as an OV5-type star.

Acknowledgments. This article is partially supported by the Bulgarian National Science Foundation under contract F-704/1997. SAZh acknowledges the financial support by the NSF grant AST-9528256.

\section{References}

Hillier, D.J. 1987 ApJS 63, 965

Hiltner, W.A. 1945, ApJ 101, 356

Ivanov, M.M., Valchev, T.S., Georgiev L.N., Barba R., Iliev, I.Kh. 1999, RevMexA\&A in press

Lamontagne, R., Moffat, A.F.J., Drissen, L., Robert, C., Matthews, J.M. 1996, AJ 112, 2227

Marchenko, S.V., Moffat, A.F.J., Eenens, P.R.J. 1998, PASP 110, 1416 